\title{
Síndrome de encefalopatía posterior reversible en paciente con diálisis peritoneal. Reporte de caso
}

\author{
Alex Altamirano Calderón (DD $\square^{1}$ and Susana Naranjo Vásconez (iD) 1 \\ ${ }^{1}$ Servicio de Neurología, Hospital General Puyo, Pastaza, Ecuador.
}

Cómo citar: Altamirano Calderón AA, Naranjo Vásconez S. Síndrome de encefalopatía posterior reversible en paciente con diálisis peritoneal. Reporte de caso. Rev. Colomb. Nefrol. 2021; 8(1):e443. https://doi.org/10.22265/acnef.8.1.443

Recibido:

23/Abr/2020

Aceptado:

03/ago/2020

Publicado:

25/Ene/2021

\section{Resumen}

Se presenta el caso de una paciente de 66 años con antecedente de enfermedad renal crónica desde hace 8 años y en terapia con diálisis peritoneal, quien acudió al servicio de urgencias por presentar deterioro del estado de conciencia asociado a convulsiones y emergencia hipertensiva. La paciente fue diagnosticada con síndrome de encefalopatía posterior reversible y luego de instaurado el tratamiento indicado la sintomatología cedió; después de un mes de evolución se observó resolución de las lesiones halladas en la resonancia magnética. Esta patología se asocia principalmente a hipertensión arterial severa de rápida instauración en correlación con diversas entidades clínicas como lo fue la hipertensión arterial en el presente caso.

Palabras clave: Síndrome de leucoencefalopatía posterior, convulsiones, hipertensión, diálisis peritoneal.

\section{Abstract}

We present the case of a 66-year-old patient with a history of chronic kidney disease for 8 years, on peritoneal dialysis therapy, who went to the emergency service for presenting deterioration in the state of consciousness associated with seizures, and hypertensive emergency, after treatment the symptoms subside and resolution of the lesions found on the MRI after one month of evolution, classified as posterior reversible encephalopathy syndrome. Considering this diagnosis in patients with chronic kidney injury associated with arterial hypertension.

Keywords: Posterior Leukoencephalopathy Syndrome, seizures, hypertension, peritoneal dialysis.

\section{$\square$ Correspondencia:}




\section{Reversible posterior encephalopathy syndrome in a patient with peritoneal dialysis. Case report}

\section{Introducción}

El síndrome de encefalopatía posterior reversible (PRES, por su sigla en inglés) es un síndrome clínico-radiológico descrito inicialmente por Hinchey et al. [1] en 1996 en una serie de casos de 15 pacientes con diferentes patologías y manifestaciones clínicas quienes presentaron lesiones encefálicas reversibles en regiones posteriores.

Los síntomas del PRES son muy variados; sin embargo, los más frecuentes incluyen cefalea, crisis epilépticas, trastornos visuales y deterioro del nivel de consciencia. Esta entidad de asocia principalmente a hipertensión arterial severa de rápida instauración en correlación con diversas entidades clínicas; así, su relación con enfermedad renal crónica (ERC) y aguda se ha descrito en múltiples series, pese a lo cual no existe una asociación establecida con la diálisis peritoneal, por lo que los estudios publicados son escasos.

El PRES es un síndrome neurológico clínicamente heterogéneo en el cual se puede lograr la reversión completa de la sintomatología y de las lesiones cerebrales cuando el tratamiento se instaura de forma oportuna. La ERC y el fracaso renal agudo están comúnmente presentes en pacientes con PRES, además este está fuertemente asociado con condiciones que coexisten en pacientes con enfermedad renal como hipertensión, enfermedades vasculares y autoinmunes, exposición a drogas inmunosupresoras y trasplante de órganos. Por lo tanto, es importante considerarlo en el diagnóstico de pacientes con enfermedad renal y síntomas neurológicos rápidamente progresivos.

Aunque el PRES es un trastorno cada vez más reconocido y con un amplio espectro clínico de síntomas y desencadenantes establecidos, aún sigue siendo poco entendido en cuanto a su fisiopatología [2].

\section{Presentación del caso}

Paciente femenina de 66 años con antecedente de ERC estadio 5D, secundaria a hipertensión arterial, quien se encontraba en terapia de reemplazo renal mediante diálisis peritoneal desde hace 8 años con doble modalidad: manual y automatizada. La mujer recibía diálisis peritoneal continua ambulatoria con la siguiente prescripción: líquido: dextrosa 1,5\% con calcio normal, volumen: $2.000 \mathrm{~mL}$, permanencia: 6 horas, intercambio: 1, y diálisis peritoneal 
cíclica continua con la siguiente prescripción: líquido: dextrosa 1,5\% y 2,5 \% con calcio normal, volumen: $10.000 \mathrm{~mL}$, ciclos: 4.

La paciente asistió al servicio de urgencias por presentar crisis epilépticas focales con generalización secundaria de 4 minutos de duración y disminución del nivel de consciencia asociado a emergencia hipertensiva $(200 / 120 \mathrm{~mm} \mathrm{Hg})$. Al examen físico neurológico se encontró estuporosa (Glasgow 10/15), sin focalidad neurológica, pálida y con pupilas isocóricas normorreactivas sin alteraciones adicionales, incluyendo pares craneales. Se inició manejo antiepiléptico con ácido valproico a dosis ponderal de $20 \mathrm{mg} / \mathrm{kg}$ /día y se mantuvo en tratamiento con $100 \mathrm{mg}$ de Losartán al día, $10 \mathrm{mg}$ de amlodipino y 12,5 mg de carvedilol cada 12 horas.

De igual forma, al ingreso a urgencias se inició infusión intravenosa con nitroprusiato de sodio, gracias a lo cual las cifras tensionales se redujeron de forma ligera. Luego de esto, la paciente fue remitida al servicio de medicina interna, a cargo de nefrología y neurología, donde se ordenó tomografía simple de cráneo que resultó normal, así como análisis de urea que se encontró en 95 y creatinina que fue de $8.6 \mathrm{md} / \mathrm{dL}$; los niveles electrolitos se hallaron normales. Luego de la estabilización hemodinámica y neurológica se indicó resonancia magnética nuclear simple de cráneo donde se observaron múltiples imágenes hiperintensas en sustancia blanca subcortical y en región cortical parieto-occipital izquierda compatibles con PRES (Figura 1).

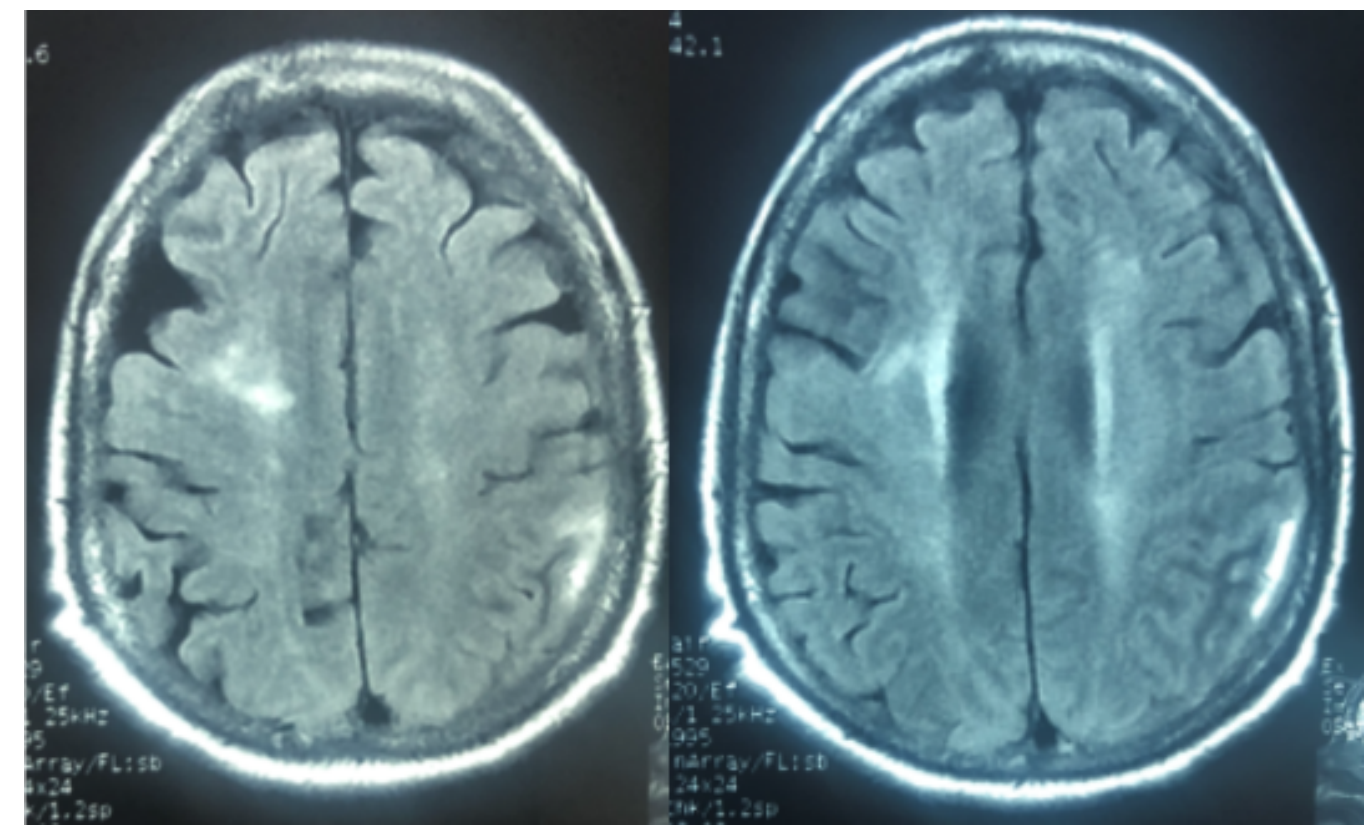

Figura 1. Resonancia magnética simple de cráneo secuencia FLAIR

Fuente: documento obtenido durante la realización del estudio. 
Durante la hospitalización se decidió también suministrarle a la paciente doxasosina a dosis de $4 \mathrm{mg} /$ día, con lo cual, de forma paulatina, recobró el nivel de consciencia sin reaparición de crisis epilépticas y con cifras tensionales de 150/80. De esta forma, fue dada de alta y se ordenó seguimiento por consulta externa a los 30 días con resonancia de control (Figura 2), donde se observó desaparición de las lesiones corticales (posibles causas de las crisis epilépticas) y disminución de estas en sustancia blanca subcortical, las cuales pudieron estar relacionadas con leucoaraiosis periventricular. Para ese entonces la paciente se encontraba asintomática.

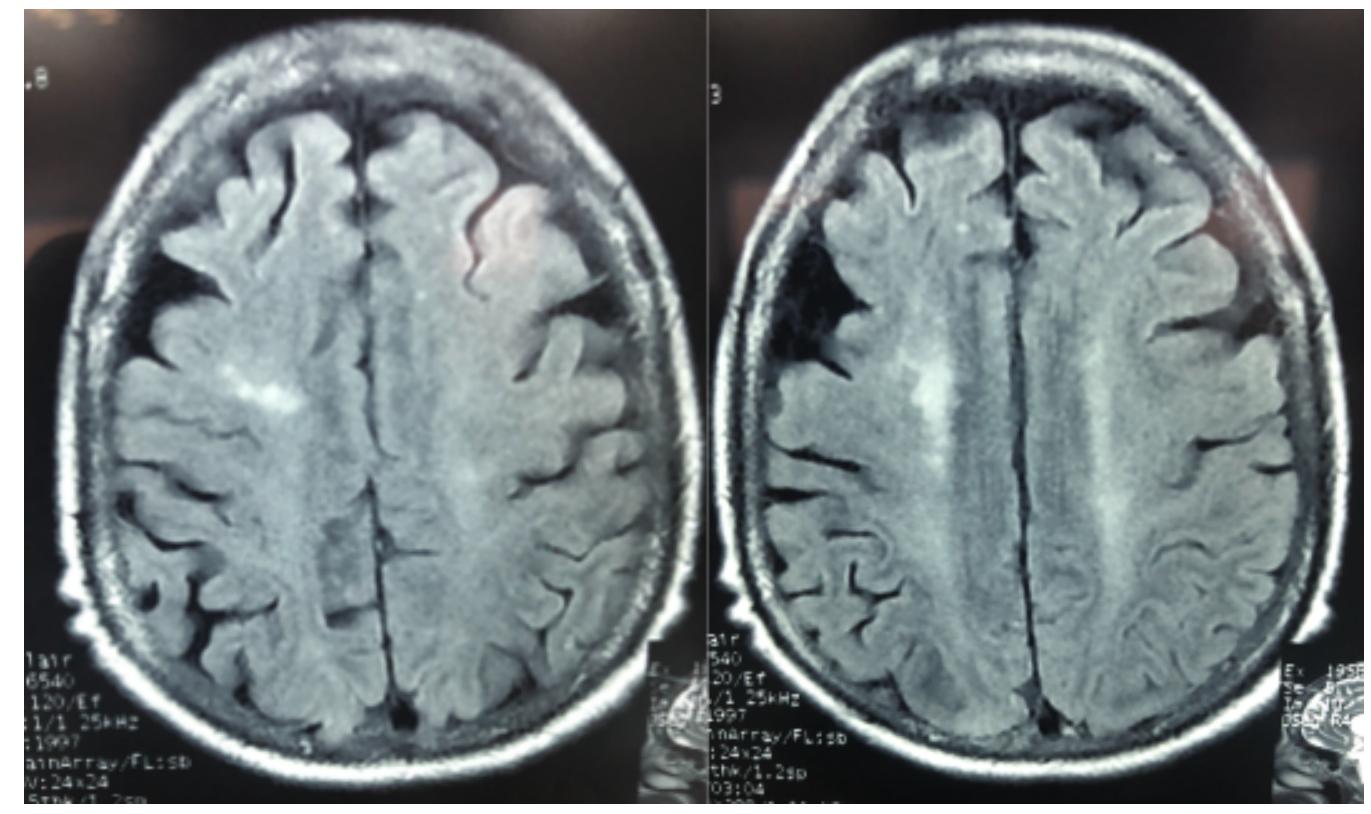

Figura 2. Resonancia magnética simple de cráneo de control

Fuente: documento obtenido durante la realización del estudio.

\section{Discusión}

Pese a que la definición del PRES implica la reversibilidad total o parcial de los hallazgos clínico-radiológicos, esto no siempre sucede y es un tema que se mantiene aún el debate [3]. Además, la fisiopatología poco establecida aumenta el enigma detrás de esta entidad, asociada inicialmente con hipoperfusión, vasoconstricción e isquemia, pues existen reportes incluso con presiones arteriales normales $[4,5]$.

De igual forma, existe una teoría alternativa de que el PRES es la consecuencia de un estado inflamatorio que provoca disfunción endotelial, lo que explicaría la asociación con procesos como sepsis, enfermedades autoinmunes o trasplantes. En estos casos la vasoconstricción provocada por el estado inflamatorio se sumaría a la inicial del mecanismo de autorregulación y la isquemia resultante sería la causa de la alteración endotelial; además, recientemente se ha 
postulado la frecuente asociación entre hipomagnesemia y PRES, y el mecanismo favorecedor del PRES de los anticalcineurínicos se ha explicado mediante esta alteración electrolítica [6].

En el presente caso la caracterización imagenológica del PRES se centró en la sustancia blanca, principalmente en regiones posteriores; sin embargo, se distinguen diferentes patrones que incluyen lóbulos frontales, temporales e incluso hemisferios cerebelosos [7].

Los datos epidemiológicos deben ser interpretados con precaución, ya que el síndrome aún puede ser infradiagnosticado debido a que la condición en ocasiones es difícil de confirmar. Esta entidad ha sido reportada en casi todos los grupos etarios, desde niños hasta adultos mayores, pero con mayor frecuencia en jóvenes o adultos de mediana edad, y por tanto la edad es un factor que puede influir en su desarrollo [8].

El PRES también se ha relacionado con hemodiálisis y ERC en múltiples series de casos [9-11]; sin embargo, su relación en pacientes con diálisis peritoneal es muy poco estudiada: existen reportes aislados, siendo algunos de ellos recurrentes en edades pediátricas, además otros han requerido manejo de la hipertensión en unidad de cuidados intensivos [12-14]. El tratamiento de esta entidad deber ser muy individualizado, siendo los antihipertensivos intravenosos como el nicardipino los medicamentos escogidos, aunque también se ha resuelto modificando la terapia a hemodiálisis [15].

Sea cual fuera el mecanismo y la resolución, la diálisis peritoneal se puede interpretar como un factor de riesgo para el desarrollo de PRES, sobre todo por la sobrecarga de volumen y la hipoalbuminemia. De igual forma, esta terapia se puede identificar como un pro-

\author{
El PRES es una entidad \\ clínica-radiológica reversible, de \\ baja incidencia y con curso \\ monofásico.
} tector relativo frente a la hemodiálisis, ya que estos pacientes poseen mayor estabilidad hemodinámica durante su terapia dialítica [16]. Estudios futuros en esta situación en particular dilucidarán su papel fisiopatológico y el adecuado tratamiento que hasta ahora no ha sido resuelto con precisión.

\section{Conclusión}

El PRES es una entidad clínica-radiológica reversible, de baja incidencia y con curso monofásico en la cual la identificación temprana optimiza el pronóstico a mediano plazo; además, el manejo multidisciplinario es el factor más relevante para disminuir la morbimortalidad asociada. 
Debido a que la asociación con diálisis peritoneal es infrecuente, no existen datos estadísticos al respecto ni estudios de seguimiento a largo plazo, de ahí la importancia de la descripción de este caso y la recomendación de una acción oportuna.

\section{Consideraciones éticas}

Los autores declaran que los procedimientos seguidos se realizaron conforme a las normas éticas del comité de experimentación humana responsable y de acuerdo con lo establecido por la Asociación Médica Mundial en la Declaración de Helsinki; que han seguido los protocolos de su centro de trabajo sobre la publicación de datos de pacientes, y que han obtenido el consentimiento informado de los pacientes y/o sujetos referidos en el artículo.

\section{Conflicto de intereses}

Ninguno declarado por los autores.

\section{Financiación}

Ninguna declarada por los autores.

\section{Agradecimientos}

Ninguno declarado por los autores.

\section{Contribución de los autores}

Alex Altamirano Calderón estuvo a cargo de la investigación, la recolección de los datos, la revisión bibliográfica y la escritura del manuscrito, y Susana Naranjo Vásconez se encargó de la escritura y revisión del documento.

\section{Referencias}

[1] Hinchey J, Chaves C, Appignani B, Breen J, Pao L, Wang A, et al. A Reversible Posterior Leukoencephalopathy Syndrome. N Engl J Med. 1996;334(8):494-500. https:/dx.doi.org/10. 1056/NEJM199602223340803. 个Ver página 2

[2] Hobson EV, Craven I, Blank SC. Posterior Reversible Encephalopathy Syndrome: A Truly Treatable Neurologic Illness. Perit Dial Int. 2012;32(6):590-4. https:/dx.doi.org/10.3747/pdi. 2012.00152. $\uparrow$ Ver página 2 
[3] Canney M, Kelly D, Clarkson M. Posterior Reversible Encephalopathy Syndrome in EndStage Kidney Disease: Not Strictly Posterior or Reversible. Am J Nephrol. 2015;41(3):177-82. https:/dx.doi.org/10.1159/000381316. $\uparrow$ Ver página 4

[4] Bartynski WS. Posterior Reversible Encephalopathy Syndrome, Part 2: Controversies Surounding Pathophysiology of Vasogenic Edema. Am J Neuroradiol. 2008;29(6):1043-9. https://x.doi.org/10.3174/ajnr.A0929. $\uparrow$ Ver página 4

[5] Iwafuchi Y, Okamoto K, Oyama Y, Narita I. Posterior Reversible Encephalopathy Syndrome in a Patient with Severe Uremia without Hypertension. Intern Med. 2016;55(1):63-8. https:/ dx.doi.org/10.2169/internalmedicine.55.5563. ^Ver página 4

[6] Chardain A, Mesnage V, Alamowitch S, Bourdain F, Crozier S, Lenglet T, et al. Posterior reversible encephalopathy syndrome (PRES) and hypomagnesemia: A frequent association? Rev Neurol https:/dx.doi.org/10.1016/j.neurol.2016.06.004. $\uparrow$ Ver página 5

[7] Bartynski WS, Boardman JF. Distinct Imaging Patterns and Lesion Distribution in Posterior Reversible Encephalopathy Syndrome. Am J Neuroradiol. 2007;28(7):1320-7. https:/ dx.doi.org/10.3174/ajnr.A0549. $\uparrow$ Ver página 5

[8] Fischer M, Schmutzhard E. Posterior reversible encephalopathy syndrome. J Neurol.

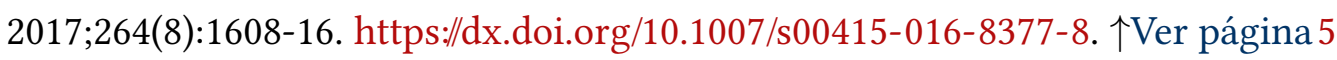

[9] Sánchez-Santana AY, Monzón-Vázquez T, Díaz-Novoa N, Valga F, Antón G, Cantón CG. Síndrome de encefalopatía posterior reversible en hemodiálisis: descripción de un caso y revisión de la literatura. Nefroplus. 2018;10(2):75-8. 个Ver página 5

[10] Vázquez Jiménez LC, Troya-Saborido MI, Bancu IE, Sol JB. Síndrome de encefalopatía posterior reversible y enfermedad renal crónica. A propósito de siete casos clínicos y revisión de la bibliografía. Nefroplus. 2016;8(1):7-13. 个Ver página 5

[11] Ganesh K, Nair RR, Kurian G, Mathew A, Sreedharan S, Paul Z. Posterior Reversible Encephalopathy Syndrome in Kidney Disease. Kidney Int Rep. 2018;3(2):502-7. https:

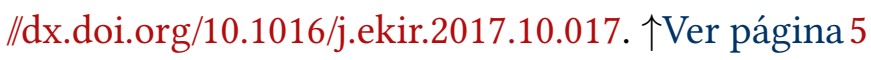

[12] Girişgen İ, Tosun A, Sönmez F, Özsunar Y. Recurrent and atypical posterior reversible encephalopathy syndrome in a child with peritoneal dialysis. Turk J Pediatr. 2010;52(4):4169. $\uparrow$ Ver página 5

[13] Graham BR, Pylypchuk GB. Posterior reversible encephalopathy syndrome in an adult patient undergoing peritoneal dialysis: a case report and literature review. BMC Nephrol. 2014;15(1):10. https:/dx.doi.org/10.1186/1471-2369-15-10. $\uparrow$ Ver página 5 
[14] Ohsawa I, Nishitani T, Fukuda H, Takeda Y, Matsuzaki K, Nagamachi S, et al. Occurrence of Reversible Posterior Leukoencephalopathy Syndrome in a Continuous Ambulatory Peritoneal Dialysis Patient. J Nephrol Ther. 2013;3(1). https://dx.doi.org/10.4172/2161-0959. 1000130. $\uparrow$ Ver página 5

[15] Kaneko S, Hirai K, Minato S, Yanai K, Mutsuyoshi Y, Ishii H, et al. A case of posterior reversible encephalopathy syndrome in a patient undergoing automated peritoneal dialysis. CEN Case Rep. 2019;8(3):178-82. https://dx.doi.org/10.1007/s13730-019-00389-1. 个Ver página 5

[16] Moreiras-Plaza M, Fernández-Fleming F, Azkárate-Ramírez N, Nájera-de la Garza W, Martín-Baez I, Hernansanz-Pérez M. Diálisis peritoneal: ¿un factor de riesgo o de protección para la encefalopatía posterior reversible (PRES)? Revisión de la literatura. Nefrología. 2018;38(2):136-40. https://x.doi.org/10.1016/j.nefro.2017.03.026.个Ver página 5 